\title{
Multilevel Inverter Topology Using Current Path Change for Zero Current Switching
}

\author{
Tomoya Sugimoto $^{* a)}$ Student Member, Takahiro Nozaki* Member \\ Toshiyuki Murakami* Fellow
}

(Manuscript received May 4, 2018, revised Sep. 4, 2018)

\begin{abstract}
In motor drive applications, a high switching frequency is required to mitigate current harmonics, which cause a copper loss. However, the high switching frequency makes the switching loss large. Hence, some switching loss reduction techniques have been studied in the past. These techniques have some problems. This paper proposes a multilevel inverter topology for switching loss reduction. Because of provision of multiple current paths, the proposed inverter realizes the zero current switching. Consequently, a loss of the whole system including an inverter and motor can be reduced with a higher switching frequency. The switching loss of the proposed inverter is smaller than that of the conventional one. It was verified that the proposed inverter has some advantages at high switching frequency, through calculations.
\end{abstract}

Keywords: multilevel inverter, switching loss, bidirectional switch, topology, zero current switching

\section{Introduction}

In motor drive applications, mitigating current harmonics is very important. The current harmonics cause a torque ripple and copper loss. Therefore, multilevel inverters are better than two level inverters. Multilevel inverters can reduce voltage stress across semiconductor switches which connect other switches in series. Thus, multilevel inverters have other advantages such as reducing $d v / d t$, voltage total harmonic distortion and electromagnetic interference. These characteristics are suitable for motor drive applications.

In general terms, multilevel inverters are categorized into three types by their topologies; Neutral Point Clamped (NPC), Flying Capacitor (FC) and Cascaded H-Bridge (CHB). The NPC inverters are used for high power AC motor drive applications, which have been studied to increase efficiency of trains ${ }^{(1)(2)}$. However, they have a disadvantage of the charge imbalance of capacitors, which need complicated modulation methods or extra circuits ${ }^{(3)(4)}$. The CHB inverters are used for very high power applications which demand power quality; static var compensator and distributed generations using renewable energy ${ }^{(1)(5)}$. However, they require multiple isolated DC voltage sources ${ }^{(6)}$. By using fuel cells and photovoltaic cells, the problem can be overcome ${ }^{(7)}$. The FC inverters are used for high bandwidth high switching frequency applications ${ }^{(1)}$. However, they require numerous capacitors. As the number of voltage levels increase, the large number of capacitors and the complex voltage balancing methods are required ${ }^{(6)}$. Additionally, a generalized

a) Correspondence to: Tomoya Sugimoto. E-mail: sugimoto@ sum.sd.keio.ac.jp

${ }^{*}$ Keio University

3-14-1, Hiyoshi, Kohoku-ku, Yokohama, Kanagawa 223-8522, Japan
Table 1. Comparison between multilevel inverters

\begin{tabular}{ccccc}
\hline & P2 & FC & NPC & Proposed \\
\hline Voltage stress of switches & $\frac{1}{N-1}$ & $\frac{1}{N-1}$ & $\frac{1}{N-1}$ & $\frac{4}{N+1}$ or $\frac{8}{N+1}$ \\
Number of switches & $(N-1) N$ & $2(N-1)$ & $2(N-1)$ & $2(4 N-13)$ \\
Diode: $2(N-2)$ & $2(4 N)$ \\
Number of capacitors & $\frac{1}{2}(N-1) N$ & $N-2$ & $N-1$ & $\frac{1}{2}(N-1)$ \\
Voltage self balancing & itself & $\times$ & $\times$ & itself \\
Voltage stress of capacitors & $\frac{1}{N-1}$ & $\frac{1}{N-1}-\frac{N-2}{N-1}$ & $\frac{1}{N-1}$ & $\frac{1}{N-2}$ \\
\hline
\end{tabular}

multilevel (P2) inverter topology was proposed ${ }^{(8)}$. FC inverters and NPC inverters can be derived from that inverter which can lead some new structure. Although the generalized multilevel inverter can balance the capacitor voltage, it requires numerous capacitors and switches.

In addition, a higher switching frequency is required in PWM drive in order to mitigate a copper loss. However, the higher switching frequency increases a switching loss of inverters ${ }^{(9)}$. Therefore, techniques to reduce the switching loss have been studied. The soft switching technology has been used to reduce the switching loss. It uses a LC resonance to keep a voltage or current of a switch zero during the entire turn-on and turn-off transition. Nevertheless, it has some limitations; a limited drive range, increase of conduction loss, and more complicated circuit ${ }^{(10)(11)}$.

The proposed topology has some advantages to the conventional topologies, which are described in Table 1. Although the voltage stress of switches to DC link voltage and the number of switches are larger than those of conventional topologies, the proposed topology can naturally balance capacitor voltage. Moreover, it does not need extra circuits for the zero current switching. This paper proposes a multilevel inverter topology using current path change for the zero current switching to reduce the switching loss. When the output voltage changes, the current path and the switch condition 
change at different timing by using multiple current paths, which realizes zero current switching.

This paper is organized as follows. In section 2, the principle of proposed inverter is presented. In section 3, the power loss is compared with the FC inverter by calculation. CHB inverters do not work with single DC voltage source and NPC inverters have a lot of diodes which cause large switching loss. For these reasons, these topologies are excepted in the comparison. Finally, the conclusions are presented in section 4.

\section{Proposed Topology}

2.1 General Topology Figure 1 illustrates the proposed inverter topology, where $n$ is the number of capacitors of the upper side or the lower side. All the capacitors are charged at the half of DC source voltage $V_{\mathrm{dc}}$ without extra circuits to balance the capacitor voltage. It has a charge-pump structure. The number of capacitors connecting to DC source voltage in series changes the output voltage which has $2 n+1$ steps. Its output voltage has $2 n+1$ steps. Assuming that the DC source is constant, the larger number of output voltage steps, the larger voltage the inverter can output. When the output voltage is changed to different level, the order of switching must be considered to prevent from short-circuit. The voltage across each capacitor is $V_{\mathrm{dc}} / 2$ and the voltage across each switch is $V_{\mathrm{dc}} / 2$ or $V_{\mathrm{dc}}$.

2.2 Current Path of Seven Level Inverter The most basic proposed inverter is five level topology. However, considering the higher level extension, the proposed seven level topology which is illustrated in Fig. 2 is suitable. Hereinafter, seven level topology is discussed. Figure 3 illustrates the switch combinations on each voltage level $V_{\text {out }}$. Behavior of capacitors of seven level topology is described by the following.

- For voltage level $0 \mathrm{~V}$, all capacitors are connecting to DC source in parallel and charged at $1 / 2 V_{\mathrm{dc}}$. Figure 2(a) illustrates this conduction path. Voltage stresses of $S_{1}, S_{2}$, $S_{5}, S_{6}, S_{10}$, and $S_{11}$ are $1 / 2 V_{\mathrm{dc}}$.

- For voltage level $1 / 2 V_{\mathrm{dc}}$, all capacitors are connecting to DC source in parallel and charged at $1 / 2 V_{\mathrm{dc}}$. Figures 2(b), (c) illustrate these conduction paths. Voltage stresses of $S_{1}, S_{2}, S_{5}$, and $S_{6}$ are $1 / 2 V_{\mathrm{dc}}$. Those of $S_{10}$ or $S_{11}$ are $V_{\mathrm{dc}}$.

- For voltage level $V_{\mathrm{dc}}, C_{11}$ and $C_{21}$ are charged. $C_{12}$ is discharging. Figure 2(d) illustrates this conduction path. Voltage stresses of $S_{1}, S_{2}, S_{7}, S_{8}$, and $S_{9}$ are $1 / 2 V_{\mathrm{dc}}$. Those of $S_{6}$ and $S_{11}$ are $V_{\mathrm{dc}}$.

- For voltage level $3 / 2 V_{\mathrm{dc}}, C_{11}$ and $C_{12}$ are discharging. Figure 2(f) illustrates this conduction path. Voltage stresses of $S_{3}, S_{4}, S_{7}, S_{8}$, and $S_{9}$ are $1 / 2 V_{\mathrm{dc}}$. Those of $S_{2}, S_{6}$, and $S_{11}$ are $V_{\mathrm{dc}}$.

Capacitors symmetrically work up side down in the cases that output voltage is negative. Theoretically, switches are classified to two groups by these maximum voltage stresses. The classification is discussed in section 3 .

2.3 Principle of Zero Current Switching Realized by Current Path Change How to realize the zero current switching is explained. When the output voltage of the proposed topology changes, four MOSFETs relate the change of the current path. The order of switching is determined in

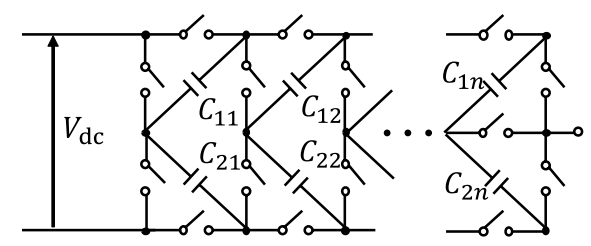

Fig. 1. Proposed topology phase-leg

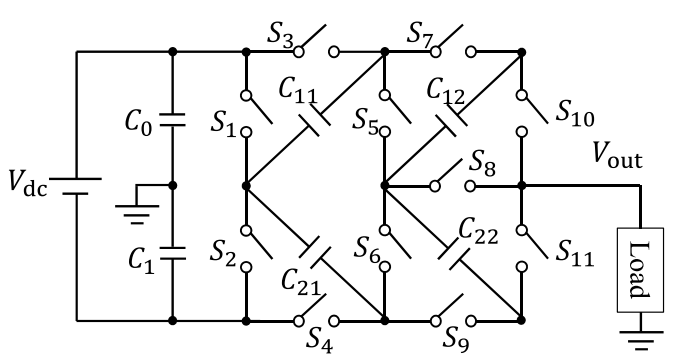

Fig. 2. Proposed single-phase seven level topology
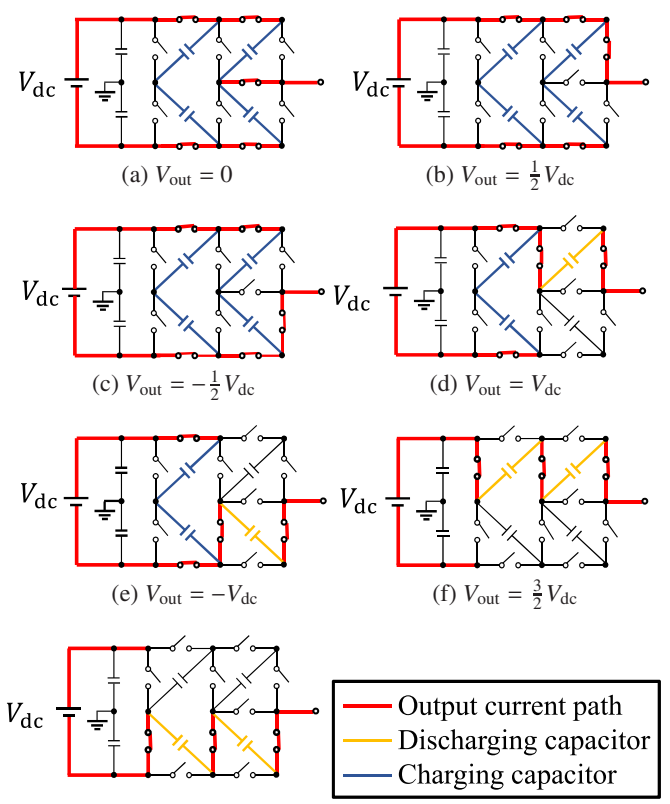

(g) $V_{\text {out }}=-\frac{3}{2} V_{\mathrm{dc}}$

Fig. 3. Current paths patterns of output voltage



Fig. 4. Bidirectional switch

order to prevent capacitors from short circuit and keep the current continuous. Hence, switches must be bidirectional switches which can control a current direction consists of two MOSFETs illustrated in Fig. 4. $S_{\mathrm{AB}}$ consist of two MOSFETs, $S_{\mathrm{A}}$ and $S_{\mathrm{B}}$ which are used for the following explanation. $S_{\mathrm{CD}}$ is the same manner to $S_{\mathrm{AB}}$. In Fig. 4, a left MOSFET can control the current flowing to the right and a right MOSFET can control the current flowing to the left. In the seven level inverter, switches are classified as illustrated in Table 2. The order of switching is classified to four cases by the current direction and the voltage change. Except for $S_{8}$, 
Table 2. Switch classification

\begin{tabular}{c|c|c}
\hline Change of output voltage & $S_{\mathrm{AB}}$ & $S_{\mathrm{CD}}$ \\
\hline$\frac{3}{2} V_{\mathrm{dc}} \Leftrightarrow V_{\mathrm{dc}}$ & $S_{3}$ & $S_{1}$ \\
$V_{\mathrm{dc}} \Leftrightarrow \frac{1}{2} V_{\mathrm{dc}}$ & $S_{7}$ & $S_{5}$ \\
$\frac{1}{2} V_{\mathrm{dc}} \Leftrightarrow 0$ & $S_{8}$ & $S_{10}$ \\
$0 \Leftrightarrow-\frac{1}{2} V_{\mathrm{dc}}$ & $S_{8}$ & $S_{11}$ \\
$-\frac{1}{2} V_{\mathrm{dc}} \Leftrightarrow-V_{\mathrm{dc}}$ & $S_{9}$ & $S_{6}$ \\
$-V_{\mathrm{dc}} \Leftrightarrow-\frac{3}{2} V_{\mathrm{dc}}$ & $S_{4}$ & $S_{2}$ \\
\hline
\end{tabular}
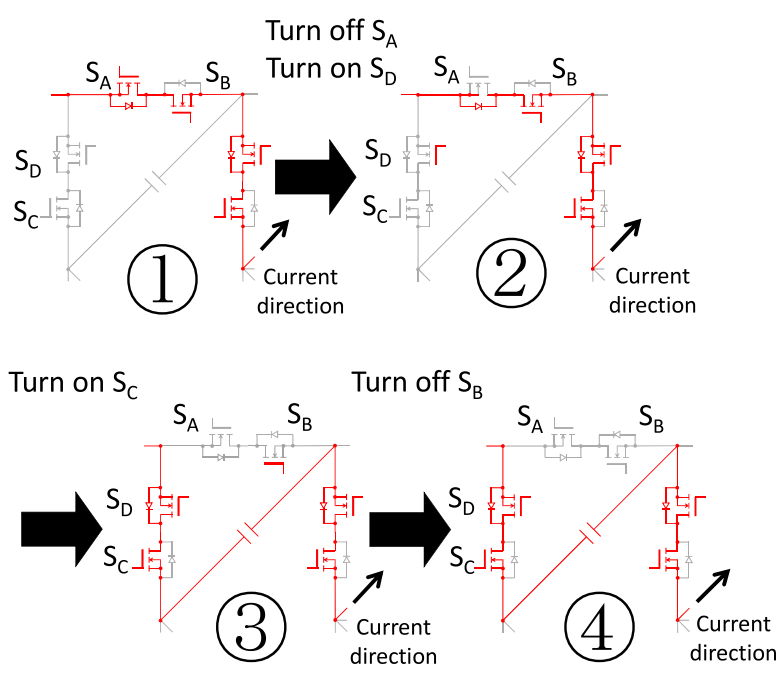

Fig. 5. Current path change when current is positive and voltage gets larger
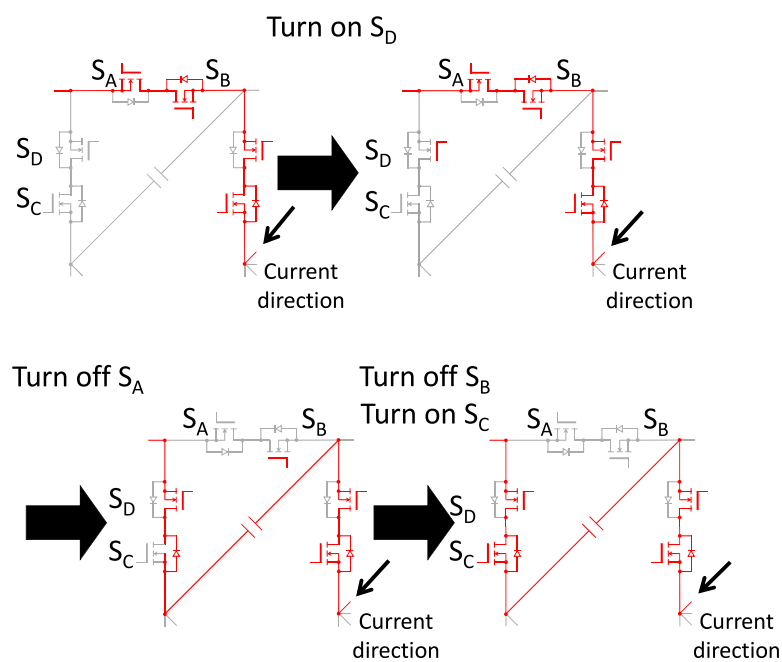

Fig. 6. Current path change when current is negative and voltage gets larger

the cases when the output voltage get larger is explained as follows. In other cases, switches work in reverse order.

Figure 5 illustrates a case that the current is positive and the voltage gets larger. Firstly, $S_{\mathrm{A}}$ turns off and $S_{\mathrm{D}}$ turns on simultaneously. The current through $S_{\mathrm{A}}$ keeps flowing, which does not cause the turn-off loss. The current is not flowing through $S_{\mathrm{D}}$, which does not cause the turn-on loss. Secondly, $S_{\mathrm{C}}$ turns on. Then, a body diode of $S_{\mathrm{A}}$ turns off and the current path changes from one passing through $S_{\mathrm{A}}$ and $S_{\mathrm{B}}$ to one passing through $S_{\mathrm{C}}$ and $S_{\mathrm{D}}$. That turn-off causes a reverse recovery loss. Finally, $S_{\mathrm{B}}$ turns off, which does not cause the turn-off loss as the current is not flowing through $S_{\mathrm{B}}$.

Figure 6 illustrates a case that the current is negative and the voltage gets larger. Firstly, $S_{\mathrm{D}}$ turns on. The current is
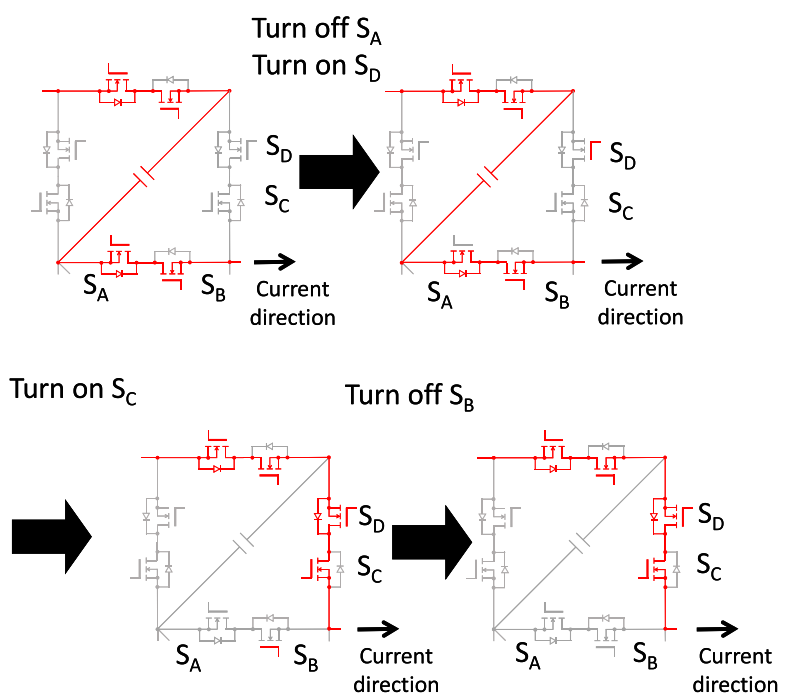

Fig. 7. Current path change when current is positive and voltage gets larger in the case including $S_{8}$

Turn on $S_{D}$
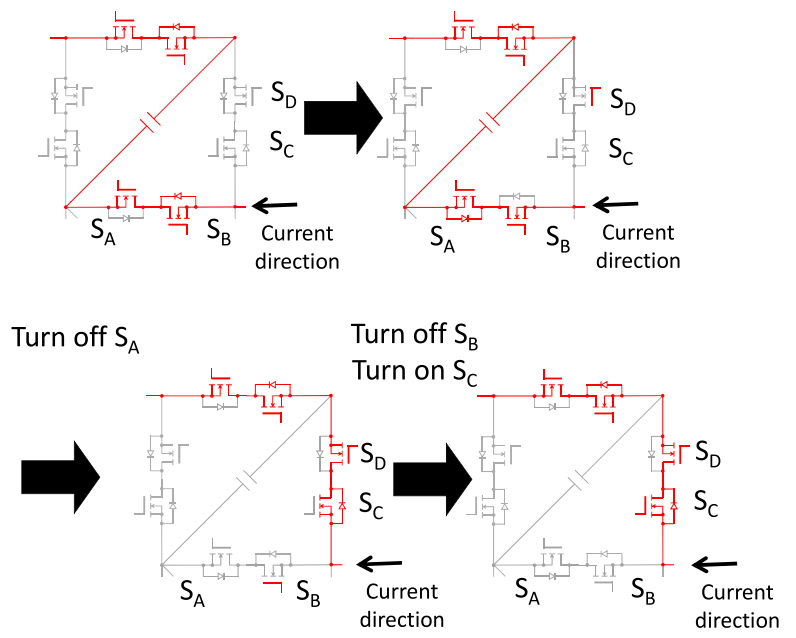

Fig. 8. Current path change when current is negative and voltage gets larger in the case including $S_{8}$

not flowing through $S_{\mathrm{D}}$, which does not cause the turn-on loss. Secondly, $S_{\mathrm{A}}$ turns off and the current path changes from one passing through $S_{\mathrm{A}}$ and $S_{\mathrm{B}}$ to one passing through $S_{\mathrm{C}}$ and $S_{\mathrm{D}}$. Finally, $S_{\mathrm{B}}$ turns off and $S_{\mathrm{C}}$ turns on simultaneously. The current is not flowing through $S_{\mathrm{B}}$, which does not cause the turn-off loss. The current of $S_{\mathrm{C}}$ keeps flowing, which does not cause the turn-off loss.

The relation between the generalized explanation and the actual switches $\left(S_{1}-S_{12}\right)$ behavior is illustrated in Table 2 . The cases including $S_{8}$ is shown in Figs. 7, 8. Although the positional relation of $S_{\mathrm{A}}, S_{\mathrm{B}}, S_{\mathrm{C}}$, and $S_{\mathrm{D}}$ is different from the former, switches similarly work. Although the current direction is opposite to top leg, the bottom leg works similarly, whose switches are classified in Fig. 9. In all cases, $S_{\mathrm{B}}$ and $S_{\text {D }}$ realize the zero current switching. Although the reverse recovery loss is caused, a sum of these losses are reduced.

The actual output voltage and current are illustrated in Fig. 10. Supposed a motor, the load is only an inductance.

The encircled part in Fig. 10(a) is focused on, in order to demonstrate realizing the zero current switching. The 


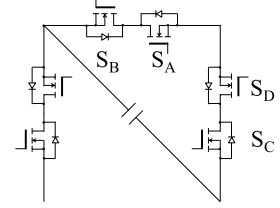

(a) Case including $S_{8}$

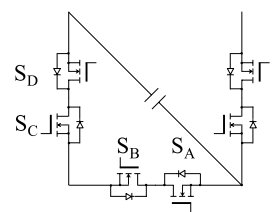

(b) Other cases
Fig. 9. Switch classification of bottom phase leg

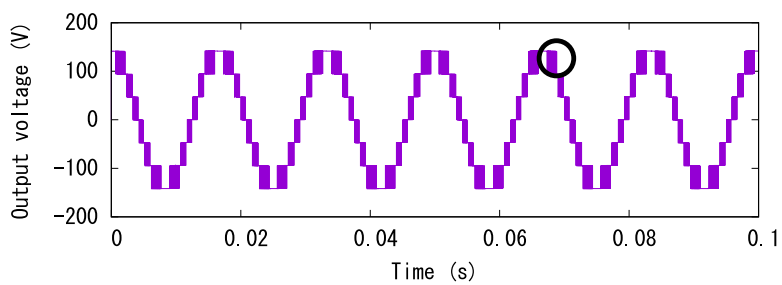

(a) Output voltage with inductive load.

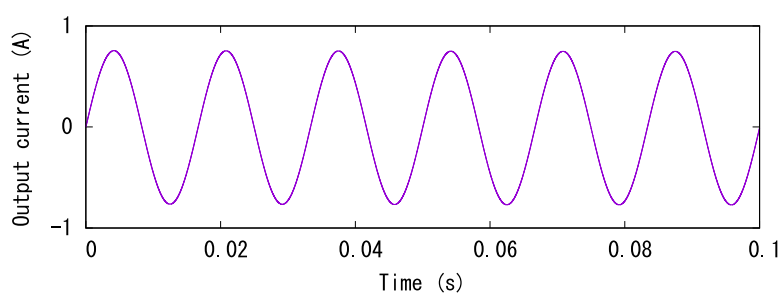

(b) Output current with inductive load

Fig. 10. Output voltage and current

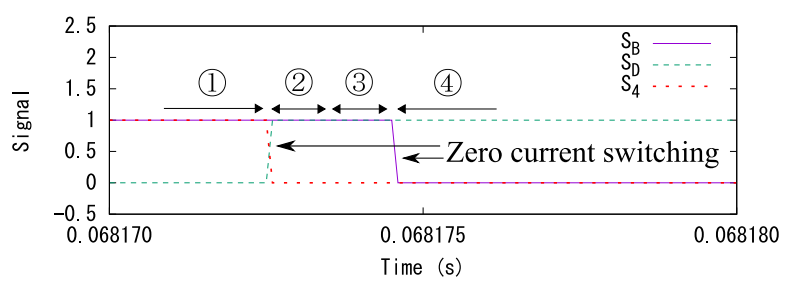

(a) Switching signal.

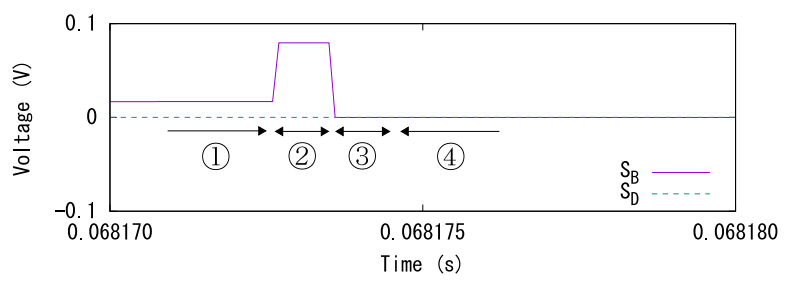

(b) Voltage across switches.

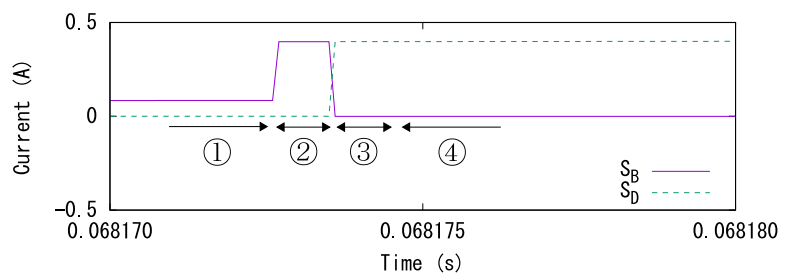

(c) Current through switches.

Fig. 11. Waveforms of $S_{\mathrm{B}}$ and $S_{\mathrm{D}}$

waveforms relating to the switches, $S_{\mathrm{B}}$ and $S_{\mathrm{D}}\left(S_{1}\right.$ and $\left.S_{3}\right)$ are illustrated in Fig. 11. The output voltage gets larger and the output current is positive. This case corresponds to the switching pattern in Fig. 11(a). When the gate signal of $S_{\mathrm{B}}$ changes to low in Fig. 11(a), the current through $S_{\mathrm{B}}$ is zero in Fig. 11(c), which does not cause the switching loss. When the gate signal of $S_{\mathrm{D}}$ changes to high in Fig. 11(a), the current

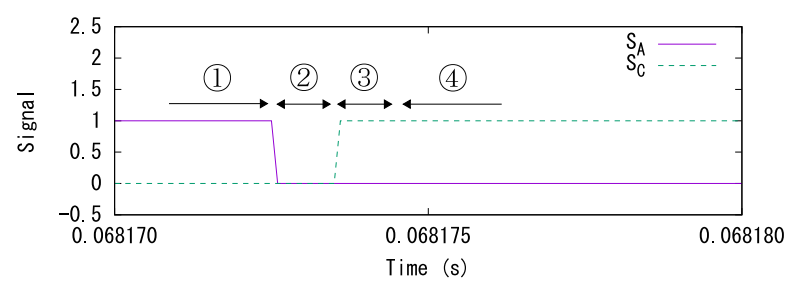

(a) Switching signal.

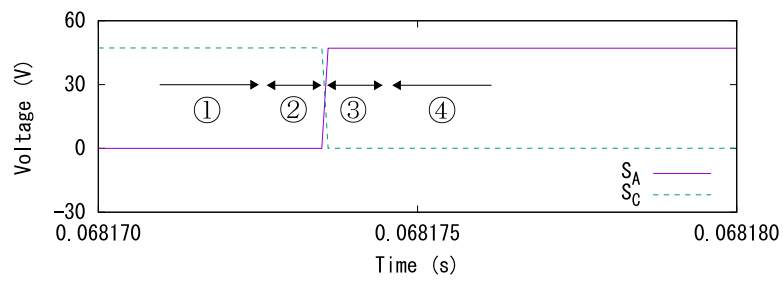

(b) Voltage across switches.

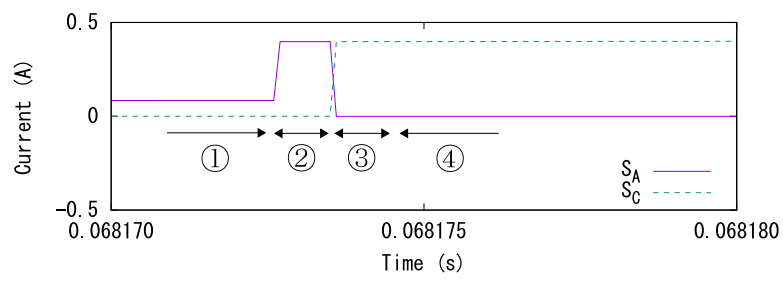

(c) Current through switches.

Fig. 12. Waveforms of $S_{\mathrm{A}}$ and $S_{\mathrm{C}}$

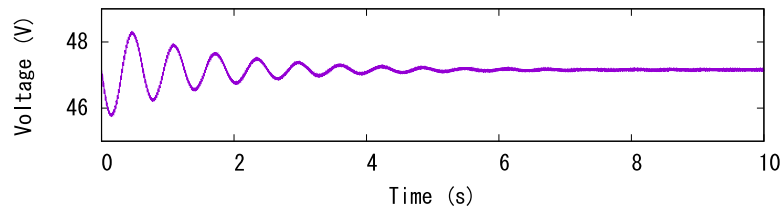

Fig. 13. Capacitor $C_{11}$ voltage

through $S_{\mathrm{D}}$ is zero in Fig. 11(c), which does not cause the switching loss. These results confirm that the zero current switching is realized in $S_{\mathrm{B}}$ and $S_{\mathrm{D}}$. The gate signal of $S_{4}$ in Fig. 2 is illustrated in Fig. 11(a). As illustrated in Fig. 3, $S_{4}$ turns off when the output voltage is changed from $V_{\mathrm{dc}}$ to $\frac{3}{2} V_{\mathrm{dc}}$. In the switching pattern explanation using Fig. 5 , the $S_{4}$ effect is not referred for simplification. Turning off $S_{4}$ makes $C_{11}$ and $C_{21}$ floating. Because of this effect, the current through $C_{11}$ and $C_{21}$ become zero, which changes the current through $S_{\mathrm{B}}$ as illustrated in Fig. 11(c).

Figure 12 illustrates waveforms of $S_{\mathrm{A}}$ and $S_{\mathrm{C}}$. After the gate signal in Fig. 12(a) become low, the voltage across


smaller in Fig. 12(c) simultaneously. This is a cause of the reverse recovery loss of $S_{\mathrm{A}}$.

Figure 13 illustrates the voltage of $C_{11}$. All the capacitors have already been charged. Compared with the voltage immediately after start, the voltage oscillation after ten second is small. Therefore, the proposed inverter can balance the capacitor voltage.

\section{Loss Analysis}

This section analyzed losses. The analysis verified that the proposed topology has a high efficiency. The loss of MOSFETs is classified to a conduction loss and a switching loss. The conduction loss is caused by on-resistance of the MOSFET. On the other hand, the switching loss is caused when 
Table 3. Analysis conditions

\begin{tabular}{cc}
\hline Output voltage $V_{0}$ & $100 \mathrm{Vrms}$ \\
Output current $I_{0}$ & $1 \mathrm{Arms}$ \\
Output voltage frequency $f$ & $60 \mathrm{~Hz}$ \\
Current phase lag & $\frac{\pi}{2}$ \\
Proposed (group1) & $2 \mathrm{SK} 3176$ \\
Proposed (group2) & $2 \mathrm{SK} 1381$ \\
FC & $2 \mathrm{SK} 2233$ \\
\hline
\end{tabular}

the MOSFET turns on or off. In addition, a reverse recovery loss is included in switching loss in this paper.

Firstly, the conduction loss is described as

$$
P_{\text {con }}=R_{\mathrm{DS}(\text { on })} I_{\mathrm{D}}^{2},
$$

where $R_{\mathrm{DS}(\text { on) }}$ and $I_{\mathrm{D}}$ are respectively on-resistance of MOSFET and drain current.

Secondly, the switching loss is a summation of turn-on loss and turn-off loss. The switching loss of the proposed inverter $P_{\text {sw_p }}$ is described as

$$
P_{\text {sw_p }}=f_{\mathrm{s}} \sum_{k=0}^{\left[\frac{f_{\mathrm{c}}}{f}\right]}\left(E_{\text {on_p }}+E_{\text {off_p }}+E_{\mathrm{d}}\right), \cdots \ldots \ldots \ldots \ldots
$$

where $f_{\mathrm{s}}, E_{\text {on_p }}$, and $E_{\text {off_p }}$ are respectively switching frequency, the turn-on loss, and the turn-off loss of the proposed inverter. On the other hand, the switching loss of the FC inverter $P_{\text {sw_f }}$ is described as

$$
P_{\text {sw_f }}=12 f_{\mathrm{s}} \sum_{k=0}^{\left[\frac{f_{\mathrm{c}}}{f}\right]}\left(E_{\text {on_f }}+E_{\text {off_f } \mathrm{f}}\right),
$$

where $E_{\text {on_f }}$ and $E_{\text {off_f }}$ are respectively the turn-on loss and the turn-off loss of the FC inverter. There is a difference of the drain-source voltage between the proposed inverter and the FC inverter, which affects the on-resistance. The onresistance of the proposed inverter is larger than that of the FC inverter. However, the number of turn-on or off times is reduced by using the proposed inverter and some switches realize the zero current switching.

In case of the seven level inverter, parameters are listed in Table 3. It is assumed that the capacitances are large enough to keep the capacitor voltage constant. Considering the output current and voltage, MOSFETs are selected. In Table 3, MOSFETs of the proposed inverter is classified with their maximum drain-source voltage. That voltage of the group2 is half as much as that voltage of the group1. The group1 includes $S_{\mathrm{C}}$ and $S_{\mathrm{D}}$. On the other hand, the group2 includes $S_{\mathrm{A}}$ and $S_{\mathrm{B}}$. The following calculations are based on a reference $^{(12)}$.

Figure 14 illustrates the conduction loss under the condition of Table 3 except for the output current. The result is calculated from Eq. (1). In Fig. 14, the conduction loss of the proposed inverter is larger than that of the FC inverter. However, the difference is small.

The switching loss of the proposed inverter decreases by $63.3 \%$ compared with the FC inverter in Fig. 15. This comparison is calculated from Eqs. (2) and (3) under the conditions of Table 3 . This result confirms that the proposed topology reduces the switching loss by realizing the zero current switching.

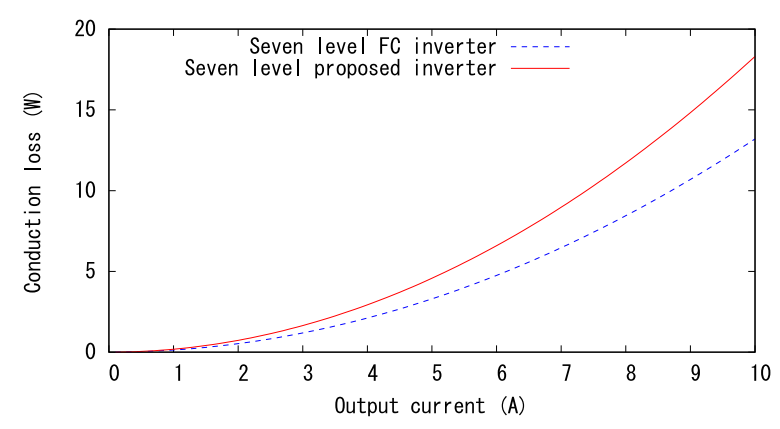

Fig. 14. Comparison of conduction loss

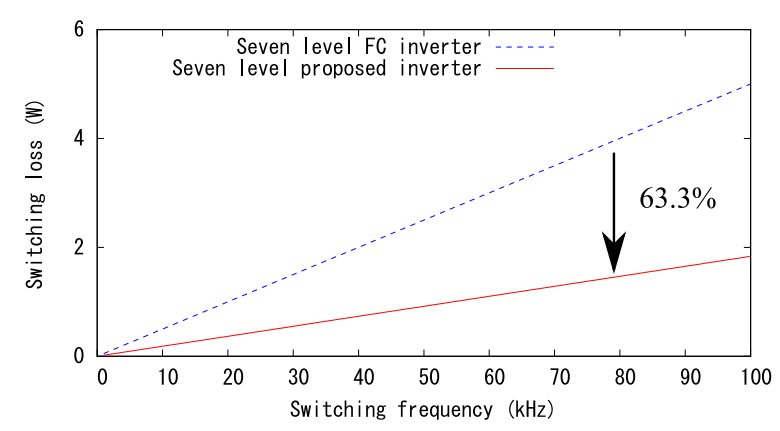

Fig. 15. Comparison of switching loss

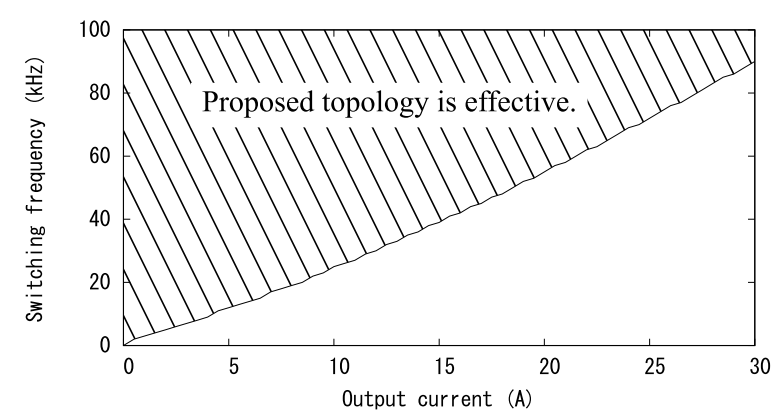

Fig. 16. Comparison of total loss

Figure 16 illustrates a total loss including the conduction loss and switching loss under the conditions of Table 3 except the output current which is calculated from Eqs. (1)-(3). Its hatched area illustrates that the total loss of the proposed inverter is smaller than that of the FC inverter. It indicates that the proposed topology is suitable for the high switching frequency PWM drive.

\section{Conclusions}

This paper proposes a multilevel inverter topology for a switching loss reduction. The proposed topology has some advantages to the conventional multilevel inverter topologies. The proposed topology does not require multiple DC sources, extra circuits to balance the capacitor voltage, and a lot of capacitors. Especially, the proposed topology itself realizes the zero current switching without extra circuits. The loss of the proposed inverter and the FC inverter are analytically compared. Consequently, compared with the FC inverter, the switching loss of the proposed inverter was reduced by $63.3 \%$. It was verified that the proposed inverter can reduce the switching loss and it has an advantage at high switching frequency. The switching loss reduction enables the switching frequency to be higher, which reduces the copper loss. 
As a result, the proposed contributes to a total loss reduction including the inverter and motor. In order to realize the usefulness, this paper proposed the original topology for the zero current switching without LC resonance.

Future work includes to reduce capacitance and to improve a limited switching frequency. Owing to the proposed topology which uses a charge pump structure, the capacitors charge and discharge repetitively with the output voltage frequency or the switching frequency. Hence, the proposed topology needs large capacitances. In addition, some steps are required when the conduction path changes, which makes the transition longer. In order to solve these problems, in the future, the switching pattern will be improved to reduce the number of steps.

\section{Acknowledgment}

This research was supported in part by the Ministry of Education, Culture, Sports, Science and Technology of Japan under Grant-in-Aid for Encouragement of Young Scientists (A), 16H06079, 2016.

\section{References}

( 1 ) L.G. Franquelo, J. Rodriguez, J.I. Leon, S. Kouro, R. Portillo, and M.A.M. Prats: "The age of multilevel converters arrives", IEEE Ind. Electron. Mag., Vol.2, No.2, pp.28-39 (2008)

( 2 ) M.Z. Youssef, K. Woronowicz, K. Aditya, N.A. Azeez, and S.S. Williamson: "Design and development of an efficient multilevel DC/AC traction inverter for railway transportation electrification", IEEE Trans. on Power Electronics, Vol.31, No.4, pp.3036-3042 (2015)

( 3 ) M. Saeedifard, R. Iravani, and J. Pou: "Analysis and control of dc-capacitorvoltage-drift phenomenon of a passive front-end five-level converter", IEEE Trans. Ind. Electron, Vol.54, No.6, pp.3255-3266 (2007)

( 4 ) D. Cui, Q. Ge, Z. Zhou, and B. Yang: "A closed-loop voltage balance method for five-level diode-clamped inverters", In Proc. of Proc. Int. Conf. IEEE IECON, pp.503-508 (2017)

( 5 ) N. Seth, V. Goel, and R.D. Kulkami: "Three phase innovative multilevel inverter topologies for research and industrial applications a review", In Proc. of Proc. Int. Conf. IEEE ICNTE (2017)

( 6 ) J. Lai and F. Peng: "Multilevel converters-a new breed of power converters", IEEE Trans. on Industry Applications, Vol.32, No.3, pp.509-517 (1996)

( 7 ) J.G. Shankar, J.B. Edward, K.S. Kumar, and I.J. Raglend: "A 31-level asymmetrical cascaded multilevel inverter with DC-DC flyback converter for photovoltaic system", In Proc. of Proc. Int. Conf. IEEE ICHVEPS, pp.277-282 (2017)

( 8 ) F. Peng: "A generalized multilevel inverter topology with slf voltage balancing", IEEE Trans. on Industry Applications, Vol.37, No.2, pp.611-618 (2001)
(9) K. Yamaguchi, K. Katsura, and T. Jikumaru: "Motor loss and temperature reduction with high switching frequency SiC-based inverters", In Proc. of 5 th IEEE Int. Workshop WiPDA, pp.127-132 (2017)

(10) J. Dudrik and J. Oetter: "High-frequency soft-switching DC-DC converters for voltage and current DC power sources", Acta Polytechnica Hungarica, Vol.4, No.2, pp.29-46 (2007)

(11) T.W. Ching and K.U. Chan: "Review of soft-switching techniques for highfrequency switched-mode power converters", In Proc. of Proc. Int. Conf. IEEE VPPC (2008)

(12) J. Brown: "Power MOSFET basics: Understanding gate charge and using it to assess switching performance", Retrieved Dec. 7, 2017, from https://www.vishay.com/docs/73217/an608a.pdf

Tomoya Sugimoto (Student Member) received the B.E. degrees in

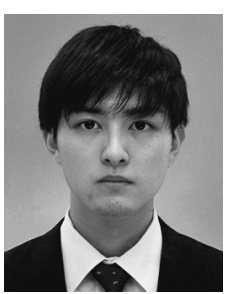
ystem design engineering from Keio University, Yokohama, Japan, in 2018. He is currently a graduate student in Keio University.

Takahiro Nozaki (Member) received the B.E. degree in system de-


sign engineering and the M.E. and Ph.D. degrees in integrated design engineering from Keio University, Yokohama, Japan, in 2010, 2012, and 2014, respectively. He was with Yokohama National University, Yokohama, Japan, from 2014 to 2015 as a Research Associate. He is currently with Keio University, Yokohama, Japan, as an Assistant Professor, and also with the Kanagawa institute of Industrial Science and Technology, Kawasaki, Japan, as a Researcher.

Toshiyuki Murakami (Fellow) received the B.E., M.E., and Ph.D. de-

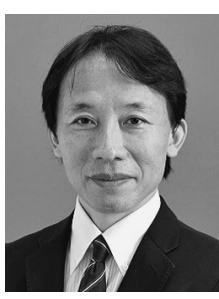
grees in electrical engineering from Keio University, Yokohama, Japan, in 1988, 1990, and 1993, respectively. In 1993, he joined the Department of Electrical Engineering, Keio University, where he is currently a Professor in the Department of System Design Engineering. From 1999 to 2000, he was a Visiting Researcher with The Institute for Power Electronics and Electrical Drives, Aachen University of Technology, Aachen, Germany. His research interests are robotics, intelligent vehicles, mobile robots, and motion control. 